\title{
Ueber eine Transformation von Luigi Bianchi.
}

\author{
(Von A. V. BäckLUND, in Lund.)
}

\begin{abstract}
In einer von der Französischen Academie der Wissenschaften gekrönten Preisschrift, die in Mémoires des Savants étrangers, t. 34 (1909) unter dem Titel «Mémoire sur la théorie des transformations des surfaces applicables sur les quadriques générales » veröffentlicht wurde, hat Bianch eine äusserst bemerkenswerte Flächentransformation aufgestellt. Auch wenn man von der grossen Wichtigkeit der erzielten Ergebnisse absehen wollte, würde auf jedẹn Fall seine dort befolgte Beweisführung ihrer ansehnlichen Tragweite wegen die grösste Aufmerksamkeit auf sich lenken. Sie ladet nicht nur zu Verallgemeinerungen ein, sondern eröffnet recht eigentlich Wege dazu, wodurch viele Punkte des Auseinandergesetzten in noch hellerem Lichte hervortreten. Das Folgende enthält einige Betrachtungen in dieser Richtung. Vornehmlich habe ich mich jedoch auf die Frage beschränkt, inwieweit für die Flächen zweiter Ordnung Bianchis Transformation erweitert werden könnte, und dabei gefunden, dass sie gewissermassen in jenem Bereiche den Charakter grösster Allgemeinheit besitzt.
\end{abstract}

I.

Bemerkungen üBer W-Kongruenzen.

1. Wenn die gemeinsame Tangentenschar zweier Flächen $S$ und $S^{\prime}$ eine $W$-Kongruenz ausmacht, korrespondieren, wie bekannt, diese $S$ und $S^{\prime}$ hinsichtlich der Berührungspunkte der gemeinsamen Tangenten so mit einander, dass jede eine infinitesimale Verbiegung gestattet, die als Verschiebung ihrer Punkte betrachtet an jedem Punkte parallel zur Normalen der anderen Fläche im korrespondierenden Punkte erfolgt. Um dies analy- 
tisch zu formulieren, denke ich mir die eine H'läche, es sei $S$, von zwei Kurvenscharen $u=C, v=C^{\prime}$ derart durchzogen, dass sie jeden Punkt dieser Fläche als Schnittpunkt zweier Kurven, und zwar einer von jeder Schar, unzweideutig darstellen, und denke mir nachher die korrespondierenden Punkte auf $S^{\prime}$ und damit auch die zu $u=C, v=C^{\prime}$ korrespondierenden Kurven derselben $S^{\prime}$ gezeichnet und analytisch durch dieselben Gleichungen $u=C, v=C^{\prime}$ wie jene von $S$ gegeben. Irgend ein Paar korrespondierende Punkte auf $S$ und $S^{\prime}$ sind dann als Punkte $(u v)$ zu bezeichnen, dieselben $u$ - und $v$-Werte für beide Punkte angenommen. Es seien ferner $x, y, z$; $x^{\prime}, y^{\prime}, z^{\prime}$ ihre Koordinaten in einem festen rechtwinkligen Cartesischen Axensysteme; dann haben wir offenbar $x, y, \ldots, z^{\prime}$ als ganz bestimmte nur von der Gestalt und der Lage von $S$ und ' $S^{\prime}$ abhängige Funktionen von $u, v$ zu betrachten, und es gelten die Gleichungen:

$$
\begin{aligned}
& x^{\prime}-x=l \frac{\partial x}{\partial u}+m \frac{\partial x}{\partial v}, \\
& y^{\prime}-y=l \frac{\partial y}{\partial u}+m \frac{\partial y}{\partial v}, \\
& z^{\prime}-z=l \frac{\partial z}{\partial u}+m \frac{\partial z}{\partial v},
\end{aligned}
$$

die überdies $l, m$ als Funktionen von $u, v$ definieren. Aus diesen Gleichungen im Verein mit denen für die zweiten Differentialquotienten von $x, y, z$, die lauten:

$$
\begin{aligned}
& \frac{\partial^{2} x}{\partial u^{2}}=\left\{\begin{array}{c}
1 \\
1
\end{array} \mid \frac{\partial x}{\partial u}+\left\{\begin{array}{c}
1 \\
2
\end{array}\right\} \frac{\partial x}{\partial v}+D X,\right. \\
& \frac{\partial^{2} x}{\partial u \partial v}=\left|\begin{array}{c}
1 \\
1
\end{array}\right| \frac{\partial x}{\partial u}+\left|\begin{array}{c}
12 \\
2
\end{array}\right| \frac{\partial x}{\partial v}+D^{\prime} X, \\
& \frac{\partial^{2} x}{\partial v^{2}}=\left\{\begin{array}{c}
22 \\
1
\end{array} \mid \frac{\partial x}{\partial u}+\left\{\begin{array}{c}
22 \\
2
\end{array} \mid \frac{\partial x}{\partial v}+D^{\prime \prime} X,\right. \text { usw., - }\right.
\end{aligned}
$$

d. h. den Gleichungen (I), S. 88 der Vorlesungen über Differentialgeometrie von Luigr Bianchi, zweite Auflage, 1910, mit den Christoffelschen Symbolen $\left|\begin{array}{c}i k \\ l\end{array}\right|$ und den Koëffizienten $D, D^{\prime}, D^{\prime \prime}$ der zweiten Fundamentalform der Fläche $S$, - leiten wir, wie es BlANGHI zuerst getan hat, für die ersten 
Differentialquotienten von $x^{\prime}, y^{\prime}, z^{\prime}$ die folgenden Formeln her:

wobei

$$
\left.\begin{array}{l}
\frac{\partial x^{\prime}}{\partial u}=L \frac{\partial x}{\partial u}+M \frac{\partial x}{\partial v}+\left(D l+D^{\prime} m\right) X, \\
\frac{\partial x^{\prime}}{\partial v}=P \frac{\partial x}{\partial u}+Q \frac{\partial x}{\partial v}+\left(D^{\prime} l+D^{\prime \prime} m\right) X, \text { usw., }
\end{array}\right\}
$$

$$
\begin{aligned}
& \left.\left.L=\frac{\partial l}{\partial u}+\left\{\begin{array}{cc}
1 & 1 \\
1
\end{array}\right\} l+\left\{\begin{array}{cc}
1 & 2 \\
1
\end{array}\right\} m+1, M=\frac{\partial m}{\partial u}+\mid \begin{array}{cc}
1 & 1 \\
2
\end{array}\right\} l+\mid \begin{array}{cc}
1 & 2 \\
2
\end{array}\right\} m,
\end{aligned}
$$

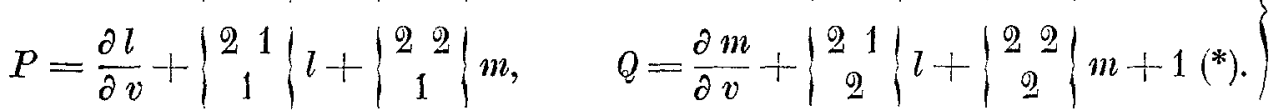

Dies war eine Folge der Berührung der Verbindungslinie der beiden Punkte $(u v)$ mit $S$; aus ihrer Berührung mit $S^{\prime}$ folgt:

$$
x-x^{\prime}=l^{\prime} \frac{\partial x^{\prime}}{\partial u}+m^{\prime} \frac{\partial x^{\prime}}{\partial v}, \text { usw., }
$$

woraus nach Einführung der Werle (2) von $\partial x^{\prime} / \partial u, \partial x^{\prime} / \partial v$ und nach Vergleichung mit (1) sich sofort ergibt:

also auch :

$$
\left.\begin{array}{c}
l^{\prime}(L Q-M P)=-l Q+m P, \\
m^{\prime}(\quad)=\quad l M-m L, \\
l^{\prime}\left(D l+D^{\prime} m\right)+m^{\prime}\left(D^{\prime} l+D^{\prime \prime} m\right)=0,
\end{array}\right\}
$$

$$
\left(D l+D^{\prime} m\right)(m P-l Q)=\left(D^{\prime} l+D^{\prime \prime} m\right)(m L-l M)\left({ }^{* *}\right) .
$$

Diese Gleichung dürfen wir dann als Bedingungsgleichung der Berülırung der Tangente $(l, m)$ von $S$ im Punkle $(u v)$ mit noch einer zweiten Fläche $S^{\prime}$ im korrespondierenden Punkte $(u v)$ auffassen, und zwar ist dies ganz unabhängig von der Natur letzterer Fläche und davon ob ihre mit $S$ gemeinsame Tangentenschar eine W-Kongruenz ist oder nicht.

2. Nunmehr mag aber rorausgesetzt sein, dass die Tangentenschar $\left(S S^{\prime}\right)$ eine $W$-Kongruenz ausmacht, dass also die Fläche $S^{\prime}$ eine infinitesimale

(*) Branchl's oben zitierte Differentialgeometrie, $\$ 284$.

$\left(^{*}\right)$ Das ist die Gleichung $(10)$ in Bianchis Théorie des Transformations des surfaces applicables sur les quadriques générales. Mém. des Sav. ëtrang., t. 34, p. 24. 
Transformation

$$
\left.\begin{array}{c}
\delta x^{\prime}=\varepsilon^{\prime} X, \quad \delta y^{\prime}=\varepsilon^{\prime} Y, \quad \delta z^{\prime}=\varepsilon^{\prime} Z, \\
\left(\varepsilon^{\prime} \text { Funktion von } u, v\right),
\end{array}\right\}
$$

zulässt, die zugleich eine Verbiegung wird, also für jedes vom Punkte $(u v)$ aus gerechnete infinitesimale Bogenelement $\left(d x^{\prime} d y^{\prime} d z^{\prime}\right)$ ron $S^{\prime}$ die Bedingung erfüllt:

oder

$$
\delta\left(d x^{\prime 2}+d y^{\prime 2}+d z^{\prime 2}\right)=0
$$

$$
d x^{\prime} d \delta d x^{\prime}+d y^{\prime} d \delta y^{\prime}+d z^{\prime} d \delta z^{\prime}=0 .
$$

Durch Einführung der Werte (6) von $\delta x^{\prime}, \delta y^{\prime}, \delta z^{\prime}$ finden wir hieraus :

$$
\varepsilon^{\prime}\left(d x^{\prime} d X+d y^{\prime} d Y+d z^{\prime} d Z\right)+d \varepsilon^{\prime}\left(X d x^{\prime}+Y d y^{\prime}+Z d z^{\prime}\right)=0,
$$

oder mit Bezug auf (2):

$$
\begin{aligned}
\varepsilon^{\prime}\left\{\left[\left(L \frac{\partial x}{\partial u}\right.\right.\right. & \left.\left.\left.+M \frac{\partial x}{\partial v}\right) d u+\left(P \frac{\partial x}{\partial u}+Q \frac{\partial x}{\partial v}\right) d v\right]\left[\frac{\partial X}{\partial u} d u+\frac{\partial X}{\partial v} d v\right]+\cdots\right\}+ \\
& +\left(\frac{\partial \varepsilon^{\prime}}{\partial u} d u+\frac{\partial \varepsilon^{\prime}}{\partial v} d v\right)\left[\left(D l+D^{\prime} m\right) d u+\left(D^{\prime} l+D^{\prime \prime} m\right) d v\right]=0
\end{aligned}
$$

oder nach wiederholter Einführung der Koëffizienten $D, D^{\prime}, D^{\prime \prime}$ der zweiten Fundamentalform von $S$ :

$$
-\Sigma d x d X=D d u^{2}+2 D^{\prime} d u d v+D^{\prime \prime} d v^{2}
$$

(BIANCHI a. a. O., § 46) :

$$
\begin{aligned}
&-\varepsilon^{\prime}\left\{\left(D L+D^{\prime} M\right) d u^{2}+\left(D P+D^{\prime}(L+Q)+D^{\prime \prime} M\right) d u d v+-\right.\left.+\left(D^{\prime} P+D^{\prime \prime} Q\right) d v^{2}\right\}+ \\
&+\frac{\partial \varepsilon^{\prime}}{\partial u}\left\{\left(D l+D^{\prime} m\right) d u^{2}+\left(D^{\prime} l+D^{\prime \prime} m\right) d u d v\right\}+ \\
&+\frac{\partial \varepsilon^{\prime}}{\partial v}\left\{\left(D l+D^{\prime} m\right) d v d u+\left(D^{\prime} l+D^{\prime \prime} m\right) d v^{2}\right\}=0
\end{aligned}
$$

Es soll aber, wie eben in anderen Worten gesagt wurde, diese Gleichung für beliebige Werte von $d u, d v$ gültig sein, daher auch jeder der Koëfti- 
zienten von $d u^{2}, d v^{2}, d u d v$ verschwinden, also:

$$
\left.\begin{array}{c}
\frac{1}{\varepsilon^{\prime}} \frac{\partial \varepsilon^{\prime}}{\partial u}=\frac{D L+D^{\prime} M}{D l+D^{\prime} m}, \\
\frac{1}{\varepsilon^{\prime}} \frac{\partial \varepsilon^{\prime}}{\partial v}=\frac{D^{\prime} P+D^{\prime \prime} Q}{D^{\prime} l+D^{\prime \prime} m}, \\
\left(D l+D^{\prime} m\right)^{2}\left(D^{\prime} P+D^{\prime \prime} Q\right)+\left(D^{\prime} l+D^{\prime \prime} m\right)^{2}\left(D L+D^{\prime} M i\right)- \\
-\left(D l+D^{\prime} m\right)\left(D^{\prime} l+D^{\prime \prime} m\right)\left(D P+D^{\prime}(L+Q)+D^{\prime \prime} M\right)=0 .
\end{array}\right\}
$$

Nach Potenzen von $l$ und $m$ geordnet und nach Unterdrückung eines Faktors $D D^{\prime \prime}-D^{\prime 2}$ nimmt letztere Gleichung die Form an:

$$
l^{2}\left(D Q-l^{\prime} M\right)-l m\left(D P-D^{\prime}(L+Q)+D^{\prime \prime} M\right)+m^{2}\left(D^{\prime \prime} L-D^{\prime} P\right)=0
$$

und diese Gleichung deckt sich völlig mit der obigen Gleichung (5).

Es kommt aber noch die oben auf $\varepsilon^{\prime}$ gestellte Forderung hinzu, dass $\varepsilon^{\prime}$ nur $u, v$ als Variablen enthalten soll, was nach (7) ergibt

$$
\frac{\partial}{\partial v}\left(\frac{D L+D^{\prime} M}{D l+D^{\prime} m}\right)=\frac{\partial}{\partial u}\left(\frac{D^{\prime} P+D^{\prime \prime} Q}{D^{\prime} l+D^{\prime \prime} m}\right)
$$

Durch diese Gleichung und die Gleichung (5) sind folglich $l, m$ zu bestimmen, um von $S$ zu einer Fläche $S^{\prime}$ der verlangten Art zu führen. Aus den Gleichungen (1) erhält man dann offenbar durch Elimination von $u$ und $v$ die Gleichung derjenigen Fläche $S^{\prime}$ in $x^{\prime}, y^{\prime}, z^{\prime}$-Koordinaten, der eine gefundene Lösung $l, m$ angehört.

3. Die weitere Rechnung gestaltet sich verhältnismässig einfach, wenn die Haupttangentenkurven von $S$ als Parameterkurven $i, v$ ausgewählt werden. Dann kommt nämlich

$$
D=0, \quad D^{\prime \prime}=0,
$$

und die Gleichungen (5) und (8) ergeben

$$
\begin{aligned}
m(m P-l Q) & =l(m L-l M), \\
\frac{\partial}{\partial v}\left(\frac{M}{m}\right) & =\frac{\partial}{\partial u}\left(\frac{P}{l}\right) .
\end{aligned}
$$


Bemerken wir noch, dass jetzt, wo $D=D^{\mu}=0$, sich notwendig ergibt

$$
\begin{aligned}
& \left|\begin{array}{cc}
1 & 1 \\
1
\end{array}\right|=\frac{\partial}{\partial u} \log \frac{\sqrt{E G-F^{2}}}{\sqrt{\rho}}, \quad\left|\begin{array}{cc}
2 & 2 \\
2
\end{array}\right|=\frac{\partial}{\partial v} \log \frac{\sqrt{E G-F^{2}}}{\sqrt{\rho}}, \\
& \left|\begin{array}{cc}
1 & 2 \\
1
\end{array}\right|=\frac{\partial}{\partial v} \log \sqrt{\rho}, \quad\left\{\begin{array}{cc}
1 & 2 \\
2
\end{array} \mid=\frac{\partial}{\partial u} \log \sqrt{\rho},\right. \\
& \text { (die absolute Krümmung }=-1 / \rho^{2} \text { gesetzt) }
\end{aligned}
$$

und führen wir $m \zeta$ statt $l$ als unbekannte Grösse ein, so bekommen wir $\left(5^{\prime}\right)$ in der Form :

$$
\begin{aligned}
\frac{2}{m}=\frac{1}{\zeta} \frac{\partial \zeta}{\partial v}-\frac{\partial \zeta}{\partial u}+\frac{1}{\zeta}\left|\begin{array}{c}
22 \\
1
\end{array}\right| & -\frac{\partial}{\partial v} \log \frac{\sqrt{E G-F^{2}}}{\sqrt{p}}- \\
& -\zeta \frac{\partial}{\partial u} \log \frac{\sqrt{E G-F^{2}}}{\sqrt{p}}+\zeta^{2}\left|\begin{array}{cc}
1 & 1 \\
2
\end{array}\right|\left({ }^{*}\right),
\end{aligned}
$$

und mit Berücksichtigung der Werte $(3)$ von $M$ und $P$ statt $\left(8^{\prime}\right)$ die Gleichung :

$$
\frac{\partial^{2}}{\partial u \partial v} \log \zeta=\frac{\partial}{\partial v}\left(\zeta\left\{\begin{array}{cc}
1 & 1 \\
2
\end{array}\right\}\right)-\frac{\partial}{\partial u}\left(\frac{1}{\zeta}\left\{\begin{array}{c}
2 \\
1
\end{array}\right\}\right) .
$$

4. Zu einer jeden Lösung $\zeta\left(\equiv \frac{l}{m}\right)=F(u, v)$ letzterer Gleichung gehört nach (10) ein Wert $f(u, v)$ von $m$, und daraus ist, nach dem am Ende von Nr. 2 Gesagten, eine ganz bestimmte Fläche $S^{\prime}$ zu erhalten. Die Bestimmung aller zu der Fläche $S$ gehörigen Flächen $S^{\prime}$ hängt somit wesentlich von der Integration der partiellen Differentialgleichung 2. O. (11) ab. Von ihren verschiedenen Lösungen gilt indessen, wie, nach $\mathrm{C}_{A U C H Y}$, von den Lösungen jeder partiellen Differentialgleichung 2. 0 . mit zwei unabhängigen Variablen $(u, v)$ und einer unbekannten Funktion ( $\zeta)$ überhaupt, dass im allgemeinen eine beliebig genommene einfach unendliche Reihe von Werten von 'ל, $\frac{\partial \zeta}{\partial u}, u, v$, etwa:

$$
u=f(v), \quad \zeta=\varphi(v), \quad \frac{\partial \zeta}{\partial u}=\psi(v)\left(^{* *}\right)
$$

(*) Oder $\frac{2}{m}=-\zeta \frac{\partial}{\partial u} \log \zeta \sqrt{\frac{E G-F^{2}}{\rho}}-\frac{\partial}{\partial v} \log \frac{1}{\zeta} \sqrt{\frac{E G-F^{2}}{\rho}}+\zeta^{2}\left\{\begin{array}{c}1 \\ 2\end{array}\right\}+\frac{1}{\zeta}\left\{\begin{array}{c}22 \\ 1\end{array}\right\}$.

${ }^{* *} \mathrm{Zu}$ ihneu kommt eine Wertreihe für $\partial \zeta / \partial v$ aus der Gleichung:

$$
\varphi^{\prime}(v)=\psi(v) f^{\prime}(v)+\frac{\partial \zeta}{\partial v} .
$$


nur in einem Integrale $\zeta=F(u, v)$ der Gleichung enthalten sein kann. Die Wertreihen ähnlicher Art, die hiervon eine Ausnahme bilden, werden als Charakteristiken der Gleichung bezeichnet. Nun enthält die vorliegende partielle Differentialgleichung 2. 0 . (11) von zweiten Derivierten von $\zeta$ nur $\partial^{2} \zeta / d u d v$, und daher muss, nach den allgemeinen Regeln, für eine Schar der Charakteristiken $d u=0$, für die anderen $d v=0$ sein. Der Kürze wegen denke ich mir jetzt (11) unter die Form gebracht:

$$
\frac{\partial^{2} \zeta}{\partial u \partial v}=A\left(\zeta, u, v, \frac{\partial \zeta}{\partial u}, \frac{\partial \zeta}{\partial v}\right)
$$

und rede nur ron denjenigen Charakteristiken, für die $d u=0$, also $\boldsymbol{u}=$ Konst. $=C$. Mit der Gleichung $u=C^{0}$ dürfen wir eine beliebige Wert. reihe $\zeta=f(v)$ zusammenstellen, aber keine Reihe $\frac{\partial \zeta}{\partial u}$ gleich einer beliebig angenommenen Funktion von $v$, wenn die Wertreihen von $\zeta, \partial \zeta / \partial u$ mit $u=C^{0}$ zusammen eine ordinäre Stellung zu einer sie enthaltenden Lösung $\zeta=F(u, v)$ von $(a)$, also mit endlichen Werten der Derivierten von $F$, einnehmen sollen. Es muss deswegen $\partial \zeta / \partial u$ vielmehr durch angemessene Integration bestimmt werden, nämlich durch Integration der Gleichung:

$$
d\left(\frac{\partial \zeta}{\partial u}\right)=A d v
$$

wobei wir in $A$ zuvor

$$
u=C^{0}, \quad \zeta=f(v), \quad \frac{\partial \zeta}{\partial v}=f^{\prime}(v)
$$

einzutragen hätten. Und diejenigen Werte der zweiten Derivierten von $\zeta$, die zu einer Lösung $\zeta=F(u, v)$ von $(a)$ gehören und sich der folgenden Wertreihe anschliessen könnten:

$$
u=C^{0}, \quad \zeta=f(v), \quad \frac{\partial \zeta}{\partial u}=\varphi\left(v, C^{0}, C^{\prime}\right),\left[\frac{\partial \zeta}{\partial v}=f^{\prime}(v)\right],
$$

wobei $\varphi$ die allgemeine Lösung von (b) mit $C^{\prime}$ als Integrationskonstante ist, werden folgende:

$$
\frac{\partial^{2} \zeta}{\partial v^{2}}=f^{\prime \prime}(v), \quad \frac{\partial^{2} \zeta}{\partial u \partial v}=A, \quad \frac{\partial^{2} \zeta}{\partial u^{2}}=\psi\left(v, C^{0}, C^{\prime}, C^{\prime \prime}\right)
$$


wobei $\psi$ mit $C^{\prime \prime}$ als willkürlicher Konstante das Integral der Gleichung

$$
d\left(\frac{\partial^{2} \zeta}{\partial u^{2}}\right)=\left(\frac{\partial \dot{A}}{\partial u}+\frac{\partial \zeta}{\partial u} \frac{\partial A}{\partial \zeta}+A \frac{\partial A}{\partial\left(\frac{\partial \zeta}{\partial v}\right)}+\frac{\partial^{2} \zeta}{\partial u^{2}} \frac{\partial A}{\partial\left(\frac{\partial \zeta}{\partial u}\right)}\right) d v
$$

bedeutet; vorab müssen jedoch die Werte $(d)$ in diese Gleichung eingeführt werden. Wir fahren mit den Differentiationen fort, so dass wir in dieser Weise zu einem beliebigen Punkte $\left(u=C^{0}, v=v^{\circ}\right)$ einer Charakteristik (c) Werte der Derivierten noch höher, sogar beliebiger Ordnung von $\zeta$ erhalten und schliesslich so durch eine nach Potenzen von $u-C^{0}, v-v^{0}$ fortschreitende Taylorsche Reihe eine Lösung $\zeta=F(u, v)$ der gesuchten Art finden. Nach dem Vorangehenden treten aber in den Koëffizienten dieser Taylorschen Reihe Integrationskonstanten $C^{\prime}, C^{\prime \prime}, C^{\prime \prime \prime}, \ldots$ in zunehmender Anzahl auf: es gibt darum $\infty^{\infty}$ Lösungen von $(a)$, die sämtlich die Reihe (c) enthalten, unter diesen $\infty^{\infty}$, denen eine beliebige der $\infty^{1}$ Reihen $(d)$ gemeinsam ist, $\infty^{\infty}$, denen eine beliebige der $\infty^{2}$ Reihen $(d)$, (e) gemeinsam angehört, usw. $\left({ }^{*}\right)$.

Diese Sätze, auf (11) angewandt, führen offenbar zu Schlüssen über die Flächen $S^{\prime}$ :

Durch jeden Streifen des dreidimensionalen Raumes ausserhalb $S$ geht im allgemeinen eine Fläche $S^{\prime}$, aber auch nur eine. Wir können nämlich immer durch einen solchen Streifen eine Linienfläche legen, die $S$ längs einer ganzen Kurve berührt; sie ergibt zugleich für die Punkte der letzteren Kurve bestimmte Werte von $l, m$, also auch Werte von $\zeta, \partial \zeta / \partial u$, - woraus eben der Satz folgt. Nur in dem Falle dass die besprochene Kurve auf $S$ eine Haupttangentenkurve wird, erleidet der Satz eine Ausnahme. Denn denken wir an irgend eine Linienfläche, die der Fläche $S$ längs einer Haupttangentenkurve umschrieben ist. Wir finden auf dieser Linienfläche nur eine einzige Schar von Kurven, längs deren dieselbe Linienfläche von Flächen $S^{\prime}$ berührt werden kann, also ausserhalb $S$, aber auf der Linienfläche $\infty^{1}$ und nur $\infty^{1}$ Streifen, die auch zu Flächen $S^{\prime}$ als ordinäre Streifen gehören können. Es gilt von diesen Streifen ausserdem, dass jeder zugleich $z u \infty^{\infty}$ Flächen $S^{\prime}$ gehört, und dass je zwei dieser Flächen, die an einer Stelle einès dieser Streifen eine Be-

(*) Man vergleiche hierzu $\$ 2$ meiner Abhandlung: Anwendung von Sätzen ubber partielle Differentialgleichungen, etc., in B. 40 der Math. Annalen, wo die entsprechenden Sätze der Theorie der partiellen Differentialgleichungen zweiter Ordnung allgemeinster Art entwickelt worden sind. 
rührung $k$ :ter Ordnung mit einander eingehen, auch an allen anderen Stellen desselben Streifens dasselbe tun.

Aus dem ersten Satze folgt, dass die Flächen $S^{\prime}$ durch eine partielle Differentialgleichung 2. 0 . in $x^{\prime}, y^{\prime}, z^{\prime}$-Koordinaten darzustellen sind. Die Streifen, von denen der zweite Satz handelt, müssen Charakteristiken derselben sein. Nun ist uns diese Gleichung aus einer anderen Theorie wohlbekannt. Man bekommt sie nämlich einfach durch Elimination von $x, y, z$ aus der Gleichung der Fläche $S$ :

und den drei Gleichungen :

$$
z=F(x, y)
$$

$$
\left.\begin{array}{c}
p\left(x^{\prime}-x\right)+q\left(y^{\prime}-y\right)=z^{\prime}-z, \\
p^{\prime}(\quad)+q^{\prime}(\quad)=z^{\prime}-z \\
\left(x^{\prime}-x\right)^{2}+\left(y^{\prime}-y\right)^{2}+\left(z^{\prime}-z\right)^{2}=\rho p^{\prime}\left(1-\frac{\left(1+p p^{\prime}+q q^{\prime}\right)^{2}}{\left(1+p^{2}+q^{2}\right)\left(1+p^{\prime 2}+q^{\prime 2}\right)}\right)
\end{array}\right\}
$$

wobei gesetzl ist

$$
\begin{gathered}
p \operatorname{statt} \frac{\partial z}{\partial x}=F^{\prime}(x), q=F^{\prime}(y), r=\frac{\partial^{2} z}{\partial x^{2}}=\frac{\partial^{2} F}{\partial x^{2}}, \ldots, p^{\prime}=\frac{\partial z^{\prime}}{\partial x^{\prime}}, \ldots, t^{\prime}=\frac{\partial^{2} z^{\prime}}{\partial y^{\prime 2}}, \\
\frac{r t-s^{2}}{\left(1+p^{2}+q^{2}\right)^{2}}=-\frac{1}{\rho^{2}}, \frac{r^{\prime} t^{\prime}-s^{\prime 2}}{1+p^{\prime 2}+q^{\prime 2}}=-\frac{1}{\rho^{\prime 2}}\left(^{*}\right) .
\end{gathered}
$$

Die fragliche Gleichung erhält damit die Form:

$$
r^{\prime} t^{\prime}-s^{\prime 2}=F\left(z^{\prime}, x^{\prime}, y^{\prime}, p^{\prime}, q^{\prime}\right)
$$

und ihre Charakteristiken werden Haupttangentenkurven der Integralflächen.

Aus dem oben Auseinandergesetzten geht dann hervor, wie die Haupttangentenkurven auf $S$ eindeutig dergleichen Kurven auf $S^{\prime}$ entsprechen, - was für die Brennflächen der W-Kongruenzen völlig kennzeichnend ist.

5. S sei nun eine Linienfäche. Dann ist eine Schar ihrer Haupttangentenkurven als bekannt anzusehen, nämlich die geraden Erzeugenden der Fläche. Nehmen wir sie zu Parameterkurven $v=C$, so baben wir nicht nur $D=0$, sondern auch

$$
\left|\begin{array}{cc}
1 & 1 \\
2
\end{array}\right|=0
$$

(*) Siehe Bianchis Differentialgeometrie, § 174 . 
zu setzen, letzteres weil jetzt die Kurven $v=C$ geodätisch sind. Ueberdies folgt aus der ersten der drei Godazzischen Gleichungen, die ich hier sämtlich aufzeichne, da ich auch im folgenden auf sie hinweisen muss:

$$
\begin{aligned}
& \frac{\partial}{\partial v}\left(\frac{D}{\sqrt{E G-F^{2}}}\right)-\frac{\partial}{\partial u}\left(\frac{D^{\prime}}{\sqrt{E G-F^{2}}}\right)+\left\{\begin{array}{c}
22 \\
2
\end{array}\right\} \frac{D}{\sqrt{E G-F^{2}}}- \\
& -2\left\{\begin{array}{c}
1 \\
2 \\
2
\end{array}\left|\frac{D^{\prime}}{\sqrt{E G-F^{2}}}+\right| \begin{array}{c}
1 \\
2
\end{array} \mid \frac{D^{\prime \prime}}{\sqrt{E G-H^{\prime 2}}}=0\right. \\
& \frac{\partial}{\partial u}\left(\frac{D^{\prime \prime}}{\sqrt{E G-F^{2}}}\right)-\frac{\partial}{\partial v}\left(\frac{D^{\prime}}{\sqrt{E G-F^{2}}}\right)+\left\{\begin{array}{c}
22 \\
1
\end{array}\right\} \frac{D}{\sqrt{E G-F^{2}}}- \\
& -2\left\{\begin{array}{c}
1 \\
1
\end{array}\right\} \frac{D^{\prime}}{\sqrt{E G-\bar{F}^{2}}}+\left\{\begin{array}{cc}
1 & 1 \\
1
\end{array} \mid \frac{D^{\prime \prime}}{\sqrt{E G-F^{2}}}=0\right. \text {, } \\
& \frac{D D^{\prime \prime}-D^{\prime 2}}{E G-F^{2}}=-\frac{1}{\rho^{2}} \text {, }
\end{aligned}
$$

es folgt, sage ich, dass wegen (12) und weil $D=0$ :

$$
\left\{\begin{array}{c}
12 \\
2
\end{array}\right\}=\frac{\partial}{\partial u} \log \sqrt{p} \text {. }
$$

Hieraus ersehen wir aber sofort, dass, falls $S$ eine Linienfläche ist, die Gleichung (8), von der nach $\mathrm{Nr}$. 2 die Bestimmung von $S^{\prime}$ wesentlich abhängt, ein vollständiges erstes Integral besitzt. Im vorliegenden Falle folgt nämlich aus (3), dass wegen (12) und (14):

$$
\frac{M}{m}=\frac{\partial}{\partial u} \log m \sqrt{\rho},
$$

weswegen offenbar die Gleichung (8) durch die folgende zu ersetzen ist:

$$
\frac{\partial}{\partial v} \log m \sqrt{\rho}=\frac{D^{\prime} P+D^{\prime \prime} Q}{D^{\prime} l+D^{\prime \prime} m}+F(v), \quad F \text { arbiträr. }
$$

Wenn wir hierin $m$ und $\zeta(\equiv l / m)$ als unbekannte Grössen einführen und $m$ mittelst (5) aus (15) eliminieren, bekommen wir eine partielle Differentialgleichung erster Ordnung für $\zeta$, jedoch mit $F(v)$ als arbiträrer Funktion, die jetzt statt (8) die gesuchten $S^{\prime}$ liefert. Dass sich die Lösung derselben durch eine Riccatische Gleichung und nachfolgende Quadraturen erzielen lassen muss (*), geht einfach aus der Bemerkung hervor, dass die krummen Haupttan-

(*) Bianghi, a. a. 0., $\S 169$, S. 316 . 
gentenkurven der Linienfläche eben durch eine Riccatische Gleichung darzustellen sind, und dass man, wenn letztere Kurven zu Parameterkurven $u=C$ genommen werden, statt (8) die Gleichung (11) anwenden darf, die aber jetzt wegen (12) ergibt

$$
\frac{\partial \zeta}{\partial v}-\zeta F(v)+\left|\begin{array}{c}
22 \\
1
\end{array}\right|=0, F \text { arbiträr, }
$$

und zur vollständigen Lösung dieser Gleichung reichen bekamnllich Quadraturen hin.

6. Auf Flächen zweiter Ordnung angewandt nimmt die Gleichung (11) ihre einfachste Form an:

$$
\frac{\partial^{2} \log \zeta}{\partial u \partial}=0
$$

da hierbei sowohl $\left\{\begin{array}{cc}1 & 1 \\ 2\end{array}\right\}$ als $\left\{\begin{array}{c}2 \\ 2 \\ 1\end{array}\right\}$ rerschwinden. Die Flächen $S^{\prime}$ sind daher. in diessem Falle aus der Formel

$$
\frac{l}{m}=\frac{U(u)}{V(v)}
$$

abzuleiten, wenn $U, V$ arbiträre Funktionen von $u$ bez. $v$ bedeuten.

Die Gleichung (10) ergibt ferner

$$
\frac{2}{m}=-\frac{V^{\prime}(v)}{V(v)}-\frac{U^{\prime}(u)}{V(v)}-\frac{\partial}{\partial v} \log \frac{\sqrt{E G-F^{\prime z}}}{\sqrt{\rho}}-\frac{U(u)}{V(v)} \frac{\partial}{\partial u} \log \frac{\sqrt{E G-F^{*}}}{\sqrt{\rho}}\left(^{*}\right) .
$$

Ist also insbesondere die Fläche $S$ durch die folgende Gleichung in $x, y, z$ gegeben:

$$
\frac{x^{2}}{a^{2}}+\frac{y^{2}}{b^{2}}-\frac{z^{2}}{c^{2}}=1
$$

und gelten damit für die Cartesischen Koordinaten ihrer Punkte die Formeln:

$$
x=a \frac{1+u v}{u+v}, \quad y=b \frac{u-v}{u+v}, \quad z=c \frac{1-u v}{u+v},
$$

(*) Oder $\frac{2}{m}=-\frac{U}{V} \frac{\partial}{\partial u} \log U \sqrt{\frac{E G-F^{2}}{\rho}}-\frac{\partial}{\partial v} \log v \sqrt{\frac{E G-F^{2}}{\rho}}$. 
so findet man

$$
\frac{E G-F^{2}}{\rho}=\frac{4 a b c}{(u+v)^{4}}
$$

(BIANch, a. a. O. $\S 303$, oder siehe nachfolgende Nr. 15 dieser Ablandlung), und wenn wir dann in Uebereinstimmung mit (16) schreiben

$$
l=(u+v) \frac{U}{W}, \quad m=(u+v) \frac{V}{W},
$$

erkennen wir sogleich aus (17), $\operatorname{dass}(*)$ :

$$
2 W=2(U+V)-(u+v)\left(U^{\prime}+V^{\prime}\right) .
$$

Hierzu wollen wir noch bemerken, dass die Gleichungen (7) mit u, vals Parametern'der Haupttangentenkurven auf $S$ im allgemeinen von der infinitesimalen Verbiegung $\varepsilon^{\prime}$ der Fläche $S^{\prime}$ lehren:

$$
\begin{aligned}
& \frac{\partial}{\partial u} \log \frac{R^{\prime}}{m}=\zeta\left\{\begin{array}{cc}
1 & 1 \\
2
\end{array}\right\}, \\
& \frac{\partial}{\partial v} \log \frac{R^{\prime}}{l}=\frac{1}{\zeta}\left\{\begin{array}{cc}
2 & 2 \\
1
\end{array}\right\}, \quad \varepsilon^{\prime}=R^{\prime} \sqrt{p},
\end{aligned}
$$

und deshalb für das Hyperboloid (18) als Fläche $S$ einfach ergeben:

oder wegen (19):

$$
R^{\prime}=l f(u)=m \varphi(v),
$$

$$
\varepsilon^{\prime}=\varkappa \sqrt{\rho} \frac{u+v}{W}
$$

wobei $*$ eine infinitesimale Konstante ist.

II.

Mıt Vorstehendem zusammengehörender Satz ÜBer abWickelbare Flächen,

7. Wie in Nr. 1 seien $S$ und $S^{\prime}$ zwei beliebige Flächen, deren Berührungspunkte mit den gemeinsamen Tangenten als einander korrespondierend betrachtet werden. $S^{\prime}$ mag sich auf eine Kurve reduzieren: jedes Flächen.

(*) ldentität (14) bei Branchr, a. a. O., $\$ 304$. 
element, das ein Bogenelement der Kurve enthält, fungiert dabei als Flächenelement dieser Fläche $S^{\prime}$, und jede Gerade, welche die Kurve trifft, ist als Tangente derselben $S^{\prime}$ anzusehen. Gelten dann für die Bogenelemente der Kurve $\left(S^{\prime}\right)$ die Gleichungen:

$$
d x^{\prime}=\alpha\left(x^{\prime}, y^{\prime}, z^{\prime}\right) d z^{\prime}, \quad d y^{\prime}=\beta\left(x^{\prime}, y^{\prime}, z^{\prime}\right) d z^{\prime},
$$

und werden $p^{\prime}, q^{\prime},-1$ proportional den Richtungscosinus des Lotes gerechnet, das auf irgend einem am Bogenelemente $\left(d x^{\prime}, d y^{\prime}, d z^{\prime}\right)$ haftenden Flïchenelement $\left(x^{\prime} y^{\prime} z^{\prime} p^{\prime} q^{\prime}\right)$ errichtet werden kann, so ergibt sich

$$
\begin{gathered}
p^{\prime} d x^{\prime}+q^{\prime} d y^{\prime}-d z^{\prime}=0, \text { also: } \\
p^{\prime} \alpha+q^{\prime} \beta=1 .
\end{gathered}
$$

Aber wenn dieses Flächenelement $\left(x^{\prime} y^{\prime} z^{\prime} p^{\prime} q^{\prime}\right)$, als der Fläche $S^{\prime}$ angehörig betrachtet, eben mit dem Flächenelemente $(x y z p q)$ von $S\left(^{*}\right)$ korrespondieren soll, muss die Verbiadungsgerade der Punkte $(x y z)$ und $\left(x^{\prime} y^{\prime} z^{\prime}\right)$ die Fläche $S$ im ersten Punkte berühren und in die Ebene des Flächenelementes $\left(x^{\prime} y^{\prime} z^{\prime} p^{\prime} q^{\prime}\right)$ fallen, weshalb also

$$
\left.\begin{array}{rl}
p\left(x^{\prime}-x\right)+q\left(y^{\prime}-y\right) & =z^{\prime}-z, \\
p^{\prime}(\quad)+q^{\prime}(\quad) & =z^{\prime}-z .
\end{array}\right\}
$$

Diese Kolrespondenz der Flächenelemente von $S$ und $S^{\prime}$ ist im allgemeinen eindeutig; nur wenn jene Gerade $\left(x^{\prime}-x, y^{\prime}-y, z^{\prime}-z\right)$ das Bogenelement (23) enthält, also im Punkte $\left(x^{\prime}, y^{\prime}, z^{\prime}\right)$ die Kurve $\left(S^{\prime}\right)$ berührt, ist an dieser Stelle die Korrespondenz unendlich vieldeutig, indem hier das ganze Büschel der Flächenelemente durch $\left(d x^{\prime}, d y^{\prime}, d z^{\prime}\right)$ dem Flächenelemente $(x y z p q)$ entspricht.

8. Anstatt bloss einer Kurve (23) können wir diese Kurven sämtlich, oder, was auf eins herauskommt, $\infty^{1}$ beliebige Kurven

$$
\left.\begin{array}{rl}
f\left(x^{\prime}, y^{\prime}, z^{\prime}, \mu\right) & =0, \\
\varphi( & =0,
\end{array}\right\}
$$

$\mu$ eine arbiträre Konstante, als reduzierte Flächen $S^{\prime}$ der gegebenen Fläche $S$ zuordnen. In diesem Falle entspricht jedem Flächenelemente von $S$ eine ganze

$\left.{ }^{*}\right) p=F^{\prime \prime}(x), q=F^{\prime}(y)$, wenn $z=F(x, y)$ die Gleichung von $S$ ist. 
Schar von $\infty^{1}$ Flächenelementen, die an denjenigen Bogenelementen der Kurven (26) haften, die von den Schnittpunkten dieser Kurven mit der Ebene des Flächenelementes von $S$ ausgehen. Diese Korrespondenz zwischen $S$ und einfach unendlich vielen in Kurven ausgearteten Flächen $S^{\prime}$ ist dann in keiner Weise von der Korrespondenz obiger Art zwischen $S$ und irgend welchen $\infty^{1}$ Flächen $S^{\prime}$ verschieden. Die Anwendung, die im Nächstfolgenden von dieser Korrespondenz gemacht wird, ist von BranchI in seiner Differentialgeometrie von 1910 teils am Ende des $\$ 282$ in etwas weiterem Umfange vorgeschlagen und teils auch in Kap. 19-21 im Einzelnen ausgeführt worden.

9. Ist nun $S$ eine beliebige Fläche und ist mit ihr eine beliebige Schar von $\infty^{1}$ Flächen $S^{\prime}$ in feste Verbindung gebracht, und rollt $S$ mit ihrer Schar von $S^{\prime}$ in Gefolge auf einer auf sie abwickelbaren Fläche $\Sigma$, und fixiert man jedesmal im Raume $\left(x^{\prime} y^{\prime} z^{\prime}\right)$ die Lagen derjenigen $\infty^{1}$ Flächenelemente jener $S^{\prime}$, die nach dem Vorangehenden dem momentanen Berührungselemente $(x y z p q)$ von $S$ und $\Sigma$ entsprechen, so bekommt man dadurch $\infty^{3}$ Flächenelemente in Raume $\left(x^{\prime} y^{\prime} z^{\prime}\right)$, die gewissermassen dort ein Bild von $\Sigma$ abgeben können. Dasselbe wäre offenbar durch zwei partielle Differentialgleichungen erster Ordnung analytisch zu definieren, und in erster Linie hatte man danach zu untersuchen, ob diese Gleichungen $\infty^{1}$ gemeinsame Integralfächen gestatten, oder nicht. Eine Darstellung der Gleichungen in Cartesischen $x^{\prime}, y^{\prime}, z^{\prime}$ - Koordinaten würde kein übersichtliches Resultat ergeben, während dagegen der von BIanchl zur Erledigung einer speziellen Frage dieser Art in Kap. 19 seiner Differentialgeometrie eingeschlagene Weg schnell zum Ziele führt.

Wie in Nr. 1 denken wir uns $S$ von zwei Kurvenscharen $u=C, v=C^{\prime}$ durchzogen und damit durch jedes Wertepaar $u, v$ je einen Punkt auf $S$ zusammen mit allen $\infty^{1}$ mit ihm korrespondierenden Punkten auf $S^{\prime}$ eindeutig bestimmt. Für die Cartesischen Koordinaten $x^{\prime}, y^{\prime}, z^{\prime}$ dieser dem Punkte $(x, y, z)$ von $S$ entsprechenden Punkte der $\infty^{1} S^{\prime}$ können wir die Gleichungen (1) und (2) verwenden. Wir wollen nun auch die Punkte auf $\Sigma$ durch dieselben $u, v$-Parameter und die Kurven auf $\Sigma$ durch ganz dieselben Gleichungen $u=C, v=C^{\prime}$ ausdrücken, die von den Kurven $u=C, v=C^{\prime}$ auf $S$ beim Rollen von $S$ auf $\Sigma$ dort als Spuren hinterlassen werden. Dann werden die in (1) und (2) vorkommenden $\partial x / \partial u, \partial x / \partial v$ sowohl zu $S$ als auch zu $\Sigma$ gehören, da jetzt am Berührungspunkte $(x, y, z)$ dieser Flächen die Linienelemente $\sqrt{E} d u, \sqrt{G} d v$ für beide von derselben Grösse und Richtung sind. Auch $X$ ist für beide Flächen dasselbe. Die zu den Flächen $S$ und $\Sigma$ gehörigen 
Gleichungen (2) werden folglich nur in Bezug auf die Werte von $L, M, P$, $Q, D, D^{\prime}, D^{\prime \prime}$ verschieden.

Was $p^{\prime}, q^{\prime}$ anbetrifft, haben wir für sie die folgenden Gleichungen $[24,25]$ schon entwickelt:

$$
\left.\begin{array}{c}
p^{\prime} \alpha+q^{\prime} \beta=1, \\
p^{\prime}\left(x^{\prime}-x\right)+q^{\prime}\left(y^{\prime}-y\right)=z^{\prime}-z .
\end{array}\right\}
$$

Hierzu kommt nun auch sowohl für $S^{\prime}$ wie für das erwähnte Bild von $\Sigma$ :

$$
\begin{aligned}
& p^{\prime} \frac{\partial x^{\prime}}{\partial u}+q^{\prime} \frac{\partial y^{\prime}}{\partial u}=\frac{\partial}{\partial} z^{\prime} \\
& p^{\prime} \frac{\partial x^{\prime}}{\partial v}+q^{\prime} \frac{\partial y^{\prime}}{\partial v}=\frac{\partial z^{\prime}}{\partial v} .
\end{aligned}
$$

Auf diese Gleichungen, einmal auf $S$, das andere Mal auf $\Sigma$ angewandt, bezieht sich die nachfolgende Rechnung, bei der selbstverständlich auch auf die Eigenschaft der Grössen $\alpha, \beta$, irgendwie Funktionen von $x^{\prime}, y^{\prime}, z^{\prime}$ zu sein, Rücksicht genommen werden musste. Weil aber die Punkte $\left(x^{\prime}, y^{\prime}, z^{\prime}\right)$ und $(x, y, z)$ als korrespondierende Punkte dastehen und die ersteren der Flächenschar $S^{\prime}$ angehören, ihre Koordinaten also der Gleichung dieser Flächenschar und ev. den Gleichungen (26) genügen, weil aber dagegen $x, y, z$ Koordinaten der Punkte von $S$, mithin determinierte Funktionen von $u, v$ sind, haben wir zunächst $\alpha, \beta$ als gegebene Funktionen von $u, v, \mu$ zu betrachten und demnach für $\mathfrak{s}$ zu setzen:

$$
\alpha=f(u, v, \mu), \beta=\varphi(u, v, \mu),
$$

p. ein var. Parameter.

Von der Beziehung von $\Sigma$ zu den in Frage gestellten Integralflächen, die aus denjenigen $\infty^{3}$ Flächenelementen $\left(x^{\prime} y^{\prime} z^{\prime} p^{\prime} q^{\prime}\right)$ zusammengesetzt wären, die bein Rollen von $S$ auf $\Sigma$ als den Elementen letzterer Fläche entsprechend herauskommen würden, gilt es, dass jede dieser Integralflächen von je einer $S^{\prime}$ der $\infty^{2}$ beim Rollen von $S$ im Raume $\left(x^{\prime} y^{\prime} z^{\prime}\right)$ erzeugten $S^{\prime}$ - Scharen berührt wird, und zwar in einem Punkte, der mit dem momentanen Berülrungspunkte von $S$ und $\Sigma$ in genau derselben für beide in Nr. 1 angegebenen Weise korrespondiert. Die Werte der zu diesem Punkte gehörenden $\alpha$ und $\beta$ wären offenbar aus (30) nach Einführung des der betreffenden $S^{\prime}$ zukommenden Wertes von $\mu$ zu erhalten. Dieser Wert von $y$. wäre von der Form 
$F(u, v)$. Die Existenz von $\infty^{1}$ Integralflächen erwähnter Art setzt einen Wert von $\mu=F(u, v, \lambda), \lambda$ eine arb. Konstante voraus. Die oben vorgelegte Frage ist einfach eine Frage nach der Möglichkeit eines solchen Wertes von $\mu$.

10. Aus den Gleichungen (27), (28) folgt nach Elimination von $p^{\prime}, q^{\prime}$ :

$$
\left|\alpha, \quad y^{\prime}-y, \quad \frac{\partial z^{\prime}}{\partial u}\right|=0
$$

oder mit Anwendung der Gleichungen (1) und (2):

$$
\left|x, \quad l \frac{\partial y}{\partial u}+m \frac{\partial y}{\partial v}, \quad L \frac{\partial z}{\partial u}+M \frac{\partial z}{\partial v}+\left(D l+D^{\prime} m\right) Z\right|=0
$$

oder nach angemessener Reduktion und Anwendung der Gleichungen

$$
\begin{aligned}
& \Sigma\left(\frac{\partial x}{\partial u}\right)^{2}=E, \quad \Sigma \frac{\partial x}{\partial u} \frac{\partial x}{\partial v}=F, \quad \Sigma\left(\frac{\partial x}{\partial v}\right)^{2}=G: \\
& \left|\begin{array}{ccc}
\alpha \frac{\partial x}{\partial u}+\beta \frac{\partial y}{\partial u}+\frac{\partial z}{\partial u} & l E+m F & L E+M F \\
\alpha \frac{\partial x}{\partial v}+\beta \frac{\partial y}{\partial v}+\frac{\partial z}{\partial v} & l F+m G & L F+M G \\
\alpha X+\beta Y+Z & 0 & D l+D^{\prime} m
\end{array}\right|=0 .
\end{aligned}
$$

Ich schreibe diese Gleichung kürzer so:

$$
\left(D l+D^{\prime} m\right) A=(X \alpha+Y \beta+Z)\left|\begin{array}{ll}
L E+M F & l E+m F \\
L F+M G & l F+m G
\end{array}\right|
$$

wo die Determinante rechts $=\left(E G-F^{2}\right)(m L-l M)$ ist, und bemerke, dass $l, m, A, X \alpha+Y \beta+Z, E, F, G$ im Berührungspunkte von $S$ und $\Sigma$ für beide Flächen dieselben Werte besitzen; dagegen muss, mit Rücksicht auf die F'ormelı (3), wenn man für $S L, M r, \ldots, D^{\prime \prime}$ gleich $L_{0}, M_{0}, \ldots, D^{\prime \prime}{ }_{0}$ selzt, für $\Sigma$ gesetzt werden

sowie

$$
L=I_{0}+\frac{\partial l}{\partial \mu} \frac{\partial \mu}{\partial u}, \quad M=M_{0}+\frac{\partial m}{\partial \mu} \frac{\partial u}{\partial u}
$$

$$
P=P_{0}+\frac{\partial l}{\partial \mu} \frac{\partial \mu}{\partial v}, \quad Q=Q_{0}+\frac{\partial m}{\partial \mu} \frac{\partial \mu}{\partial v},
$$

Wenn dann für $\Sigma$ die Zeichen $D, D^{\prime}, D^{\prime \prime}$ reserviert werden, können wir aus (31) 
schliessen :

$$
\frac{D l+D^{\prime} m}{D_{0} l+D_{0}^{\prime} m}=\frac{m L-l M}{m L_{0}-l M_{0}}=1+\frac{m \frac{\partial l}{\partial \mu}-l \frac{\partial m}{\partial \mu}}{m L_{0}-l M_{0}} \frac{\partial \mu}{\partial u} .
$$

In ganz derselben Weise ist aus (27) und (29) und mit Berücksichtigung von (33) zu schliessen :

$$
\frac{\nu^{\prime} l+D^{\prime \prime} m}{\bar{D}_{0}^{\prime} l+D_{0}^{\prime \prime} m}=\frac{m P--l Q}{m P_{0}-l Q_{0}}=1+\frac{m \frac{\partial l}{\partial \mu}-l \frac{\partial m}{\partial \mu}}{m P_{0}-l Q_{0}} \frac{\partial \psi}{\partial v} .
$$

Wir wissen aber aus (5), dass

$$
m P_{0}-l Q_{0}=\frac{D_{0}^{\prime} l+D_{0}^{\prime \prime} m}{D_{0} l+D_{0}^{\prime} m}\left(m L_{0}--l M_{0}\right)
$$

und dürfen daher letztere Gleichung durch die folgende erselzen :

$$
\frac{\left(D^{\prime}-D_{0}^{\prime}\right) l+\left(D^{\prime \prime}-D^{\prime \prime}\right) m}{D_{0} l+D_{0}^{\prime} m}=\frac{m \frac{\partial l}{\partial y}-l \frac{\partial m}{\partial \mu}}{m L_{0}-l M I_{0}} \frac{\partial y}{\partial v},
$$

die also im Verein mit (34) zur Bestimmung von $\mu$ dienen würde.

Wenn insbesondere die Haupttangentenkuvven auf $S$ zu $u$, $v$ Kurven genommen werden, beliommen diese Gleichungen die einfachere Gestalt:

$$
\left.\begin{array}{c}
\Omega \frac{\partial \mu}{\partial u}=\frac{D l+\left(D^{\prime}-D_{0}^{\prime}\right) m}{D_{0}^{\prime} m}, \\
\Omega \frac{\partial \mu}{\partial v}=\frac{\left(D^{\prime}-D_{0}^{\prime}\right) l+D^{\prime \prime} m}{D_{0}^{\prime} m}, \quad \Omega=m^{2} \frac{\frac{\partial}{\partial \mu}\left(\frac{l}{m}\right)}{m L_{0}-l M_{0}} ;
\end{array}\right\}
$$

doch, wann werden sie unbeschrünkt integrierbar? So ist denn jetzt die oben aufgeworfene Frage nach y. als $F(u, v, \lambda) \mathrm{zu}$ formulieren.

11. Wenn, wie früher, $\zeta$ statt $l / m$ geschrieben und $m$ durch (10) in $\zeta, u, v, \partial \zeta] \partial u, \partial \zeta / \partial v$ ausgedrückt wird, $\mu$ bei diesen Differentiationen konstant gehalten, bekommen wir

$$
\left.\begin{array}{rl}
\Omega=2 \frac{\partial \zeta}{\partial \mu}: \zeta \frac{\partial}{\partial u} & \log \left(\zeta \frac{\sqrt{E G-F^{2}}}{\rho \sqrt{\rho}}\right)- \\
& -\frac{\partial}{\partial v} \log \left(\frac{1}{\zeta} \frac{\sqrt{E G-F^{2}}}{\rho \sqrt{\rho}}\right)-\zeta^{2}\left\{\begin{array}{cc}
1 & 1 \\
2
\end{array}\left|+\frac{1}{\zeta}\right| \begin{array}{cc}
2 & 2 \\
1
\end{array}\right\}
\end{array}\right\}
$$


und fassen hernach die Gleichungen (36) als Gleichungen für $\zeta$ und $\mu$ auf. Wir schreiben sie in der Form:

$$
\left.\begin{array}{l}
\Omega \frac{\partial \mu}{\partial u}=\frac{D}{D_{0}^{\prime}} \zeta+\frac{D^{\prime}}{D_{0}^{\prime}}-1, \\
\Omega \frac{\partial \mu}{\partial v}=\left(\frac{D^{\prime}}{D_{0}^{\prime}}-1\right) \zeta+\frac{D^{\prime \prime}}{D_{0}^{\prime}}
\end{array}\right\}
$$

und suchen sodann durch Differentiation die Existenzbedingungen für Lösungen der Form:

$$
\zeta=f(u, v, \mu), \quad \mu=F(u, v, \lambda), \quad \lambda \text { eine arb. Konstante, }
$$

zu erhalten. Zunächst kommt:

$$
\begin{aligned}
& \frac{\partial \Omega}{\partial v} \frac{\partial \mu_{0}}{\partial u}-\frac{\partial \Omega}{\partial u} \frac{\partial u}{\partial v}=\zeta \frac{\sqrt{E G-F^{\prime \prime}}}{D_{0}^{\prime}} \mid \frac{\partial}{\partial v}\left(\frac{D}{\sqrt{E G-F^{2}}}\right)- \\
& \left.\quad-\frac{\partial}{\partial u}\left(\frac{D^{\prime}}{\sqrt{E G-F^{2}}}\right)-\frac{D}{D_{0}^{\prime}} \frac{\partial}{\partial v}\left(\frac{D_{0}^{\prime}}{\sqrt{E G-F^{2}}}\right)+\frac{D^{\prime}}{D_{0}^{\prime}} \frac{\partial}{\partial u}\left(\frac{D_{0}^{\prime}}{\sqrt{E G-F^{2}}}\right)\right\}+ \\
& \quad+\frac{D}{D_{0}^{\prime}}\left(\frac{\partial \zeta}{\partial v}+\frac{\partial \zeta}{\partial{ }^{\prime}} \frac{\partial \mu}{\partial v}\right)-\frac{D^{\prime}-D_{0}^{\prime}}{D_{0}^{\prime}}\left(\frac{\partial \zeta}{\partial u}+\frac{\partial \zeta}{\partial \mu} \frac{\partial \mu}{\partial u}\right)+ \\
& \quad+\frac{\sqrt{E G-F^{2}}}{D_{0}^{\prime}}\left\{\frac{\partial}{\partial v}\left(\frac{D^{\prime}}{\sqrt{E G-F^{2}}}\right)-\frac{\partial}{\partial u}\left(\frac{D^{\prime \prime}}{\sqrt{E G-F^{2}}}\right)-\right. \\
& \left.\quad-\frac{D^{\prime}}{D_{0}^{\prime}} \frac{\partial}{\partial v}\left(\frac{D_{0}^{\prime}}{\sqrt{E G-F^{2}}}\right)+\frac{D^{\prime \prime}}{D_{0}^{\prime}} \frac{\partial}{\partial u}\left(\frac{D_{0}^{\prime}}{\sqrt{E G-F^{2}}}\right)\right\}
\end{aligned}
$$

wobei die vorstehenden Differentiationen von $\Omega$ und $\zeta$ in Bezug auf $u, v, \mu$ so auszuführen sind, als ob diese Variablen von einander unabhängig wären.

Zufolge der zwei ersten der Gleichungen (13), der Gleichung

$$
D D^{\prime \prime}-D^{\prime 2}=-D_{0}^{\prime 2}=-\frac{D_{t} G-F^{2}}{\rho^{2}}
$$

der Gleichungen $\left(36^{\prime}\right)$, der Werte (9) der in (13) auftretenden Symbole $\left|\begin{array}{cc}i & k \\ l\end{array}\right|$ und endlich des Wertes (37) von $\Omega$ finden wir für die obige Bedingungsgleichung 
die expliziertere Form :

$$
\begin{aligned}
& \left.\frac{D}{D_{0}^{\prime}} \zeta \mid \frac{1}{\Omega} \frac{\partial \Omega}{\partial v}-\frac{\partial}{\partial v} \log \zeta-\frac{1}{\zeta}\left\{\begin{array}{c}
22 \\
1
\end{array}\right\}+\frac{\partial}{\partial v} \log \left(\frac{\sqrt{E G-F^{2}}}{\rho \sqrt{\rho}}\right)\right\}- \\
& -\frac{D^{\prime \prime}}{D_{0}^{\prime}} \mid \frac{1}{\Omega} \frac{\partial \Omega}{\partial u}-\zeta\left\{\begin{array}{c}
1 \\
Q
\end{array} \mid+\frac{\partial}{\partial u} \log \left(\frac{\sqrt{L^{2} G-F^{2}}}{\rho \sqrt{\rho}}\right)\right\}+ \\
& +\frac{D^{\prime}-D_{0}^{\prime}}{D_{0}^{\prime}}\left|\frac{1}{\Omega} \frac{\partial \Omega}{\partial v}-\zeta \frac{1}{\Omega} \frac{\partial \Omega}{\partial u}-\frac{\partial}{\partial v} \log \zeta-\frac{1}{\zeta}\right| 2|2|+ \\
& \left.\left.+\frac{\partial}{\partial v} \log \left(\frac{\sqrt{E G-F^{2}}}{\rho \sqrt{p}}\right)+\zeta^{2} \mid 11\right\}-\zeta \frac{\partial}{\partial u} \log \left(\frac{\sqrt{E G-F^{2}}}{\rho \sqrt{\rho}}\right)\right\}=0
\end{aligned}
$$

oder in leichtverständlicher Abkürzung:

$$
A^{\zeta} D+(A-B \zeta)\left(D^{\prime}-D_{0}^{\prime}\right)-B D^{\prime \prime}=0
$$

Dies ist eine partielle Differentialgleichung Q. O. für ‘. Sie gibt diejenigen $S^{\prime}$-Scharen $\zeta=f(u, v, \mu)$, die im Stande sind, einer gegebenen auf $S$ abwickel-


chenschar $(\mu=F(u, v, i))$ zuzuorthen.

Beiläutig bemerke ich, dass, wenn $S$ eine Linienfläche ist, und $v=C$ die Gleichung ihrer geraden Erzeuggenden, finden wir für alle auf $S$ abwickelbare Linienfläclien : $D=0, D^{\prime}=D_{0}^{\prime},\left|\begin{array}{cc}1 & 1 \\ 2\end{array}\right|=0$, und werden dann in jedem Integrale $\zeta=f(u, v, \mu)$ der partiellen Differentialgleichung 1.0 .

$$
\Omega \frac{\sqrt{E G-F^{2}}}{\rho \sqrt{\rho}}=\Phi(v, \mu), \quad \Phi \text { arbiträr },
$$

die Gleichung einer Flächenschar $S^{\prime}$ erkennen, die für säntlich jene Linierflächen je eine Flächenschar $\mu=F(v, \lambda)$ liefert.

12. Wenn für eine jede auf $S$ abwickelbare Fläche das bezügliche Gleichungspaar (36') unbeschränkt integrierbar sein soll, müssen sowolıl $A$ als $B$ verschwinden, weshalb:

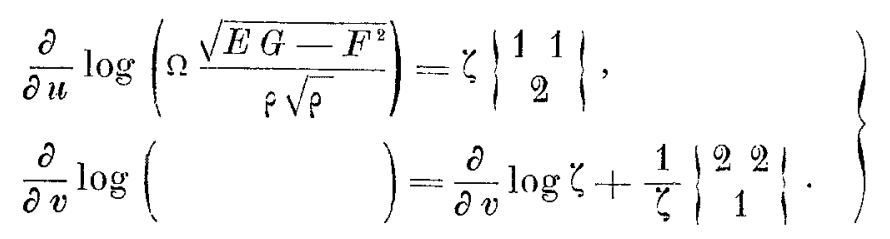


Aber das kann unmöglich so sein, wofern nicht erstens

$$
\frac{\partial^{2}}{\partial u \partial v} \log \zeta=\frac{\partial}{\partial v}\left(\zeta\left|\begin{array}{cc}
1 & 1 \\
2
\end{array}\right|\right)-\frac{\partial}{\partial u}\left(\frac{1}{\zeta}\left|\begin{array}{c}
2 \\
1
\end{array}\right|\right)
$$

und zweitens, wie aus der Zusammenstellung von (38) mit den Formeln (21) der Nr. 6 sofort einleuchtet:

$$
\Omega=\frac{\varepsilon^{\prime}}{m} \frac{\rho}{\sqrt{E G-F^{2}}} .
$$

Vorausgesetzt also, dass die Bedingungen (39) und (40) erfüllt sind, dann muss die der Fläche $S$ zugeordnete und mit ihr fest vereinigte Flächenschar $S^{\prime}$ beim Rollen von $S$ auf $\Sigma$ eine besondere Schar von $\infty^{1}$ Flächen in der am Anfange von N. 7 näher erklärten Weise umhüllen. Diese umhüllten Flächen will ich mit $\Sigma^{\prime}$ bezeichnen und bemerke, dass jene Flächen $S^{\prime}$, wenn umgekehrt $S$ in Ruhe bleibt und $\Sigma$ in fester Verbindung mit der Flächenschar $\Sigma$ ' auf $S$ rollt, in derselben Weise aus $\Sigma$ ' als eine besondere Art von Enveloppen zu erhalten sind. Es sei nun $\Sigma_{1}$ irgend cine zweite auf $S$ abwickelbare Fläche, $\Sigma_{1}^{\prime}$ eine der von $S^{\prime}$ in der oben erklärten Weise beim Rollen von $S$ auf $\Sigma_{1}$ umhüllten $\infty^{1}$ Flächen, $M$ ein auf $\Sigma_{1}$ beliebig angenommener Punkt und $b_{1}$ der hiermit korrespondierende Punkt auf $\Sigma_{1}^{\prime}$. Beim Rollen von $S$ auf $\Sigma_{1}$ wird mit $b_{1}$ der Punkt auf $S^{\prime}-a$ nemne ich ihn - zusammenfallen, der bei der gegenseitigen Berührung jener Flächen $S$ und $\Sigma_{1}$ in $M$ mit eben diesem Punkte als zu der Fläche $S$ gehörig korrespondiert. $\Sigma_{1}^{\prime}$ wird dann in diesem Punkte $a\left(b_{1}\right)$ von $S^{\prime}$ berührt. Aber auch $\Sigma$ kann, ebensowie $S$, auf $\Sigma_{1}$ rollen, und bei der einmal eintretenden gegenseitigen Berührung dieser Flächen in $M$ wird $\Sigma^{\prime}$ die Fläche $S^{\prime}$ im korrespondierenden Punkte $a$ und also auch $\Sigma^{\prime}{ }_{1}$ in demselben Punkte $a$ berühren. Hieraus folgt, dass die Flächen $\Sigma$, ebensowie sie beim Rollen von $\Sigma$ auf $S$ die Flächen $S^{\prime}$ umhüllen, beim Rollen von $\Sigma$ auf $\Sigma_{1}$ die Flächen $\Sigma_{1}^{\prime}$ umhüllen. Die Schar der $\Sigma^{\prime}$ wird sich also ganz so zu $\Sigma, \Sigma, S$ und allen anderen auf $\Sigma$ abwickelbaren Flächen verhalten, wie sich die Schar der $S^{\prime}$ zu $S, \Sigma, \Sigma_{1}$ und allen anderen auf sie abwickelbaren Flächen verhält. Nun zeigt die Bedingungsgleichung (39), weil sie sich mit der Gleichung (11) deckt, dass eine jede jener $\infty^{1}$ Flächen $S^{\prime}$ mit $S$ die Brennfäche einer $W$ - Kongruenz ausmacht. $\Sigma$ war eine beliebige der auf $S$ abwickelbaren Flächen: ein und dieselbe Flächenschar $S^{\prime}$ wird für jede $\Sigma$ in dev ervähnten Weise, oder durch Integration eines Gleichungsparres $\left(36^{\prime}\right)$, eine ähnliche Flächenschar इ' ergeben. 
Hierzu ist noch folgendes zu bemerken. Ganz so wie beim Rollen von $S$ auf $\Sigma$ im gemeinsamen Berührungspunkte beider Flächen ihre absolute Krümmung dieselhe ist, ist auch, der dritten der Gleichungen (11') in Nr. 4 zufolge, im Berührungspunkte von $S^{\prime}$ und $\mathfrak{Y}^{\prime}$ ihre absolute Krïmmung diesclbe.

13. Als eine Folge der gegenseitigen Korrespondenz der Haupttangentenkurven von $\Sigma$ und $\Sigma^{\prime}(\mathrm{Nr} .4)-$ den Fall (39), (40) stets rorausgesetzt ist zu erkennen, dass, wenn eine $\Sigma^{\prime}$-Schar aus Flächen bestände, die sämtlich zu Kurven (26) ausgeartet wären, diese Kurven eben Gerade sein müssten. Denn jede muss Leitkurve $\infty^{1}$ oskulierender Streifen sein, die denjenigen Streifen von $\Sigma$ entsprechen würden, die sich an die cine Schar der Haupttangentenkurven dieser Fläche anschliessen.

14. Bei einer anderen Gelegenheit werde ich beweisen, dass sämtliche Flächen einer $S^{\prime}$ - Schar, die den Forderungen (39) und (40) genügt, ans einer beliebigen Fläche der Schar durch wiederholte Verbiegungen parallel den Normalen von $S$ herauskommen müssen.

III.

\section{Anwendeng auf Fläghen zweiter Ordnung.}

15. Wenn die Fläche $S$ beim Rollen auf einer auf sie abwickelbaren Fläche $\Sigma$ nur $\infty^{1}$ Lagen einnimmt und dennoch mit $\Sigma$ in allen Punkten in Berührung kommt, muss diese Berührung zwischen $S$ und $\Sigma$ in jeder Lage der ersteren Fläche längs einer ganzen Kurve erfolgen, und ausserdem muss in jedem Punkte dieser Kurve die absolute Krümmung beider Flächen gleich ausfallen. Nach einem für die Theorie der Verbiegung der Flächen fundamentalen Satze muss jede solche Berührungskurve notwendig Haupttangentenkurve sein. Es würde folglich $\Sigma$ derart auf $S$ abgewickelt werden können, dass sich dabei eine Schar Haupttangentenkurven beider Flächen decken. Und nach einem Satze von Bonnex sind dann diese Haupttangentenkurven geradlinig: die Flächen $S$ und $\Sigma$ müssen Linienflächen sein.

Setzen wir dann voraus, dass es für eine Linienfläche $S$ eine Flächenschar $S^{\prime}$ obiger Art gibt, die zu einer jeden auf $S$ abwickelbaren Fläche $\Sigma$ 
eine Flächenschar $\Sigma^{\prime}$ liefert, so würde u. a. jede $\Sigma^{\prime}$ derjenigen Schar dieser Art, die zu einer Linienfläche $\Sigma$ gehört, von jeder $S^{\prime}$ längs einer Haupttangentenkurve berührt. Wenn aber die $\boldsymbol{\infty}^{1} S^{\prime}$ Linienflächen sind, deren gerade Erzeugenden mit denen von $S$ korrespondieren, so müssen auch die letzterwähnten $\Sigma$ ' Linienflächen derselben Art sein. Dies wird offenbar auch gelten, wenn die Flächen $S^{\prime}$ zu Kurven und dann notwending zu Geraden (Nr. 13) ausgeartet wären. Diesen Fall werde ich für das einschalige Hyperboloid als Fläche S vollständig behandeln.

Wir haben also jetzt die Fläche $S$ in gewöhnlichen mit ihr fest verbundenen Cartesischen Axenkoordinaten durch folgende Gleichung darzustellen:

$$
\frac{x^{2}}{a^{2}}+\frac{y^{2}}{b^{2}}-\frac{z^{2}}{c^{2}}=1
$$

und die ihr zugeordnete $S^{\prime}$ - Schar in denselben Koordinaten durch die zwei Gleichungen (26):

$$
x^{\prime}=\alpha z^{\prime}+\gamma, \quad y^{\prime}=\beta z^{\prime}+\delta,
$$

$\alpha, \beta, \gamma, \delta$ in noch unbestimmt gelassener Weise einen variablen Parameter $\mu$ enthaltend. Die hierzu gehörenden Werte von $l$ und $m$ ergeben sich aus den Gleichungen (1), die zunächst liefern:

$$
\begin{aligned}
& x-\alpha z-\gamma+l\left(\frac{\partial x}{\partial u}-\alpha \frac{\partial z}{\partial u}\right)+m\left(\frac{\partial x}{\partial v}-\alpha \frac{\partial z}{\partial v}\right)=0, \\
& y-\beta z-\delta+l\left(\frac{\partial y}{\partial u}-\beta \frac{\partial z}{\partial u}\right)+m\left(\frac{\partial y}{\partial v}-\beta \frac{\partial z}{\partial v}\right)=0,
\end{aligned}
$$

weshalb, wie nach Einführung der Werte (18) von $x, y$, zeicht ersichtlich:

$$
\left.\begin{array}{rl}
l & =\frac{u+v}{2} \frac{(b c \alpha-a \delta)\left(u^{2}-1\right)+(a b-c(\alpha \delta-\beta \gamma))\left(u^{2}+1\right)-2(a c \beta+b \gamma) u}{a b(1-u v)-b c \alpha(1+u v)-a c \beta(u-v)}, \\
m & =\frac{u+v}{2} \frac{(b c \alpha+a \delta)\left(v^{2}-1\right)+(a b+c(\alpha \delta-\beta \gamma))\left(v^{2}+1\right)+2(a c \beta-b \gamma) v}{a b(1-u v)-b c \alpha(1+u v)-a c \beta(u-v)} \cdot
\end{array}\right\}
$$

Es erhält damit $\%(\equiv l / m)$ die schon aus Nr.6 zu erwartende Form $U: V$, wobei besonders:

$$
\left.\begin{array}{l}
U=(b c \alpha-a \delta)\left(u^{2}-1\right)+(a b-c(\approx \delta-\beta \gamma))\left(u^{2}+1\right)-2(a c \beta+b \gamma) u, \\
V=(b c \alpha+a \delta)\left(v^{2}-1\right)+(a b+c(\alpha \delta-\beta \gamma))\left(v^{2}+1\right)+2(a c \beta-b \gamma) v .
\end{array}\right\}
$$


Hierdurch ist auch, wie in Nr. 6 als Folge der Identitäten

$$
\left|\begin{array}{cc}
1 & 1 \\
2
\end{array}\right|=0, \quad\left\{\begin{array}{cc}
2 & 2 \\
1
\end{array} \mid=0\right.
$$

bemerkt wurde, der Bedingung (39) Genüge geleistet. Dass dagegen die zweite Bedingung, die Bedingung (40), nicht durch beliebige Werte von $x, \beta, \gamma, \delta$ zu erfüllen ist, sehen wir bald. Wir können nämlich wegen der Formeln (19), (22) diese Bedingung so schreilsen:

$$
\Omega=* \frac{P \sqrt{p}}{\sqrt{E G-F^{2}}} \frac{u+v}{m W}=\stackrel{*}{V} \frac{P \sqrt{P}}{\sqrt{E G-F^{2}}},
$$

* eine arb. Konstante, oder unter Beachtung dessen, dass wir nach (37) zu setzen haben:

$$
\begin{aligned}
& \Omega=2 \frac{V \frac{\partial U}{\partial \mu}-U \frac{\partial V}{\partial \mu}}{V}: U^{\prime}(u)-V^{\prime}(v)-U \frac{\partial}{\partial u} \log \frac{\rho \sqrt{\rho}}{\sqrt{E G-F^{2}}}+ \\
& +V \frac{\partial}{\partial v} \log \frac{P \sqrt{\rho}}{\sqrt{E G-F^{2}}} \\
& V \frac{\partial U}{\partial \mu}-U \frac{\partial V}{\partial \mu}=\frac{x}{2} \frac{\rho \sqrt{\rho}}{\sqrt{E G-F^{2}}}\left[\frac{\partial U}{\partial u}-\frac{\partial V}{\partial v}-\right. \\
& \left.-U \frac{\partial}{\partial u} \log \frac{\rho \sqrt{\rho}}{\sqrt{E G-F^{2}}}+V \frac{\partial}{\partial v} \log \frac{\rho \sqrt{\rho}}{\sqrt{E G-F^{2}}}\right] \text {. }
\end{aligned}
$$

Was die Werte der hierin steckenden $\rho, \sqrt{E G-F^{2}}$ anbetrifft, so sind sie bekanntlich durch blosse Differentiationen der Gleichungen (18) folgendermassen zu gewinnen. Man berechnet

$$
\begin{aligned}
E=\Sigma\left(\frac{\partial x}{\partial u}\right)^{2}, \quad F=\Sigma \frac{\partial x}{\partial u} \frac{\partial x}{\partial v}, \quad G=\mathrm{\Sigma}\left(\frac{\partial x}{\partial v}\right)^{2}, \\
D_{\circ}^{\prime}=\frac{1}{\sqrt{E G-F^{2}}}\left|\frac{\partial^{2} x}{\partial u \partial v}, \frac{\partial y}{\partial u}, \frac{\partial z}{\partial v}\right|=- \\
=-4 \frac{a b c}{(u+v)^{4} \sqrt{E G-F^{2}}}=-\frac{\sqrt{E G-F^{2}}}{\rho}
\end{aligned}
$$


und bekommt dann

$$
\begin{aligned}
& \frac{\rho \sqrt{\rho}}{\sqrt{E G-F^{2}}}= \frac{(u+v)^{6}\left(E G-F^{2}\right)}{8(a b c)^{3 / 2}}= \\
&=\frac{a^{2} b^{2}(1-u v)^{2}+b^{2} c^{2}(1+u v)^{2}+c^{2} a^{2}(u-v)^{2}}{2(a b c)^{3 / 2}}, \\
& \frac{\partial}{\partial u} \log \frac{\rho \sqrt{\rho}}{\sqrt{E G-F^{2}}}=2 \frac{b^{2} c^{2} v(1+u v)-a^{2} b^{2} v(1-u v)+a^{2} c^{2}(u-v)}{b^{2} c^{2}(1+u v)^{2}+a^{2} b^{2}(1-u v)^{2}+a^{2} c^{2}(u-v)^{2}} \\
& \frac{\partial}{\partial v} \log \frac{\rho \sqrt{\rho}}{\sqrt{E G-F^{2}}}=2 \frac{b^{2} c^{2} u(1+u v)-a^{2} b^{2} u(1-u v)-a^{2} c^{2}(u-v)}{b^{2} c^{2}(1+u v)^{2}+a^{2} b^{2}(1-u v)^{2}+a^{2} c^{2}(u-v)^{2}} .
\end{aligned}
$$

Die Gleichung $\left(44^{\prime}\right)$ nimmt hierdurch die Form an :

$$
\begin{aligned}
V \frac{\partial U}{\partial \mu}-U \frac{\partial V}{\partial \mu}=C\left\{\frac{1}{2}\left(\frac{\partial U}{\partial u}-\frac{\partial V}{\partial v}\right)\left(b^{2} c^{2}(1+u v)^{2}+a^{2} b^{2}(1-u v)^{2}+a^{2} c^{2}(u-v)^{2}\right)-\right. \\
-U\left(b^{2} c^{2} v(1+u v)-a^{2} b^{2} v(1-u v)+a^{2} c^{2}(u-v)\right)+ \\
\left.+V\left(b^{2} c^{2} u(1+u v)-a^{2} b^{2} u(1-u v)-a^{2} c^{2}(u-v)\right)\right\}
\end{aligned}
$$

die sich nach Einführung der Werte (43) von $U$ und $V$ noch bedeutend vereinfacht und sich folgendermassen schreiben lässt:

$$
\begin{aligned}
& V \frac{\partial U}{\partial u}-U \frac{\partial V}{\partial \mu}=C\left\{-2 b \beta\left(a^{2}+c^{2}\right)\left(1-u^{2} v^{2}\right)+2 a c \gamma\left(u^{2}-v^{2}\right)+\right. \\
& +2 a \alpha\left(b^{2}+c^{2}\right)(u-v)(1-u v)-2 c\left(a^{2}-b^{2}\right)(u-v)(1+u v)- \\
& -2 a b(\alpha \delta-\beta \gamma)(u+v)(1-u v)-2 b c \delta(u+v)(1+u v)\}
\end{aligned}
$$
gleich

Das linke Glied dieser Gleichung wird den Gleichungen (43) zufolge

$$
\begin{aligned}
V\left(\left(b c \alpha^{\prime}-a \delta^{\prime}-c(\alpha \delta-\beta \gamma)^{\prime}\right) u^{2}-2\left(a c \beta^{\prime}+b \gamma^{\prime}\right) u-\right. & \\
- & \left.\left(b c \alpha^{\prime}-a \delta^{\prime}+c(\alpha \delta-\beta \gamma)^{\prime}\right)\right)- \\
-U\left(\left(b c \alpha^{\prime}+a \delta^{\prime}+c(\alpha \delta-\beta \gamma)^{\prime}\right) v^{2}+\right. & 2\left(a c \beta^{\prime}-b \gamma^{\prime}\right) v- \\
& \left.-\left(b c \alpha^{\prime}+a \delta^{\prime}-c(\alpha \delta-\beta \gamma)^{\prime}\right)\right),
\end{aligned}
$$


wobei abkürzungsweise $\alpha^{\prime}, \ell^{\prime}, \ldots$, statt $\frac{\partial \alpha}{\partial \mu}, \frac{\partial \beta}{\partial \mu}, \ldots$, gesetzt ist. Unter nochmaliger Anwendung der Werte (43) ron $U$ und $Y$ ergibt sich ferner hieraus:

$$
\begin{aligned}
& V \frac{\partial U}{\partial \mu}-U \frac{\partial V}{\partial \mu}=2(u+v)(1-u v) a b\left[c^{2}\left(\alpha \beta^{\prime}-\alpha^{\prime} \beta\right)-\left(\gamma^{\prime}-\gamma^{\prime} \delta\right)\right]- \\
& -2(u+v)(1+u v) b c\left[a^{2} \beta^{\prime}-\left(\gamma(\alpha \delta-\beta \gamma)^{\prime}-\gamma^{\prime}(x \delta-\beta \gamma)\right)\right]- \\
& -2(u-v)(1-u v) a\left[b^{2} \gamma^{\prime}-c^{2}\left(\beta(\alpha \delta-\beta \gamma)^{\prime}-\beta^{\prime}(\alpha \delta-\beta \gamma)\right)\right]+ \\
& +2(u-v)(1+u v) c\left[b^{2}\left(x \gamma^{\prime}-x^{\prime} \gamma\right)-a^{2}\left(\beta \delta^{\prime}-\beta^{\prime} \delta\right)\right]+ \\
& +2 u^{2} v^{2} b\left[a c\left(\alpha^{\prime} \dot{\delta}-\alpha \delta^{\prime}-(\alpha \delta-\beta \gamma)^{\prime}\right)-\right. \\
& \left.-c^{2}\left(\alpha(\alpha \delta-\beta \gamma)^{\prime}-z^{\prime}(\alpha \delta-\beta \gamma)\right)-\alpha^{2} \delta^{\prime}\right]+\cdot \\
& +9\left(u^{2}+v^{2}\right) a b c\left(\alpha \delta^{\prime}-\alpha^{\prime} \delta-(\alpha \delta-\beta \gamma)^{\prime}\right)- \\
& -2\left(u^{2}-v^{2}\right) a c\left[(\alpha \delta-\beta \gamma) \delta^{\prime}-\delta(x \delta-\beta \gamma)^{\prime}-b^{2} \alpha^{\prime}\right]- \\
& -8 a b c u v\left(\beta \gamma^{\prime}-\gamma\left(\beta^{\prime}\right)+2 a b\left(a \delta^{\prime}-c(\alpha \delta-\beta \gamma)^{\prime}\right)+\right. \\
& +2 b c^{2}\left[\alpha(\alpha \delta-\beta \gamma)^{\prime}-\alpha^{\prime}(\alpha \delta-\beta \gamma)\right]-
\end{aligned}
$$

$-2 a b c\left(\alpha \delta^{\prime}-\alpha^{\prime} \delta\right)$.

Der Forderung (40) gemäss müssen (45), (46) denselben Wert von $V \frac{\partial U}{\partial \mu}-U \frac{\partial V}{\partial \mu}$ liefern; m. a. W. die Koëffizienten derselben Kombinationen von $u$ und $v$ in den beiden Ausdrücken müssen einander gleich werden. Wir schliessen dann

aus den Koëffizienten für $u v: \beta \gamma^{\prime}-\beta^{\prime} \gamma=0$,

aus denen für $u^{2} v^{2}: a c\left(\alpha^{\prime} \delta-\delta^{\prime}-(\alpha \delta-\beta \gamma)^{\prime}\right)-$

$$
-c^{2} \alpha(\alpha \delta-\beta \gamma)^{\prime}+c^{2} \alpha^{\prime}(\alpha \delta-\beta \gamma)-a^{2} \delta^{\prime}=C\left(\alpha^{2}+c^{2}\right) \beta,
$$




$$
\text { für } \begin{gathered}
1: a c\left(\alpha^{\prime} \delta-\alpha \delta^{\prime}-(\alpha \delta-\beta \gamma)^{\prime}\right)+ \\
+c^{2} \alpha(\alpha \delta-\beta \gamma)^{\prime}-c^{2} \alpha^{\prime}(\alpha \delta-\beta \gamma)+\alpha^{2} \delta^{\prime}=-C\left(a^{2}+c^{2}\right) \beta, \\
u^{2}+v^{2}: \alpha^{\prime} \delta-\alpha \delta^{\prime}+(\alpha \delta-\beta \gamma)^{\prime}=0, \\
u^{2}-v^{2}:(\alpha \delta-\beta \gamma) \delta^{\prime}-\delta(\alpha \delta-\beta \gamma)^{\prime}-b^{2} \alpha^{\prime}=-C \gamma, \\
(u+v)(1-u v): c^{2}\left(\alpha \beta^{\prime}-\alpha^{\prime} \beta\right)-\gamma^{\prime}+\gamma^{\prime}=-C(\alpha \delta-\beta \gamma), \\
(u+v)(1+u v): \alpha^{2} \beta^{\prime}-\gamma(\alpha \delta-\beta \gamma)^{\prime}+\gamma^{\prime}(\alpha \delta-\beta \gamma)=C \delta, \\
(u-v)(1+u v): b^{2}\left(\alpha \gamma^{\prime}-\alpha^{\prime} \gamma\right)-\alpha^{2}\left(\beta \delta^{\prime}-\beta^{\prime} \delta\right)=-C\left(a^{2}-b^{2}\right), \\
(u-v)(1-u v): b^{2} \gamma^{\prime}-c^{2} \beta(\alpha \delta-\beta \gamma)^{\prime}+c^{2} \beta^{\prime}(\alpha \delta-\beta \gamma)=-C \alpha\left(b^{2}+c^{2}\right) .
\end{gathered}
$$

Aus den vier ersten Gleichungen folgt:

$$
\begin{gathered}
\gamma=K \beta, \quad K \text { konst., } \\
\alpha \delta-\beta \gamma=C^{\prime}, \quad C^{\prime} \text { konst., } \\
\delta=K^{\prime} \alpha, \quad K^{\prime} \text { konst., } \\
\left(C^{\prime} c^{2}-K^{\prime} a^{2}\right) \alpha^{\prime}=C\left(\alpha^{2}+c^{2}\right) \beta ;
\end{gathered}
$$

ferner aus den übrigen Gleichungen:

$$
\begin{gathered}
\left(C^{\prime} K^{\prime}-\cdots b^{2}\right) \alpha^{\prime}=-C K \beta, \\
\left(K K^{\prime}+c^{2}\right)\left(\alpha \beta^{\prime}-\alpha^{\prime} \beta\right)=-C C^{\prime}, \\
\left(C^{\prime} K+a^{2}\right) \beta^{\prime}=C K^{\prime} \alpha, \\
\left(K b^{2}+K^{\prime} a^{2}\right)\left(\alpha \beta^{\prime}-\alpha^{\prime} \beta\right)=-C\left(\alpha^{2}-b^{2}\right), \\
\left(C^{\prime} c^{2}+K b^{2}\right) \beta^{\prime}=-C\left(b^{2}+c^{2}\right) \propto .
\end{gathered}
$$

Die sechs letzten Gleichungen reduzieren sich auf die folgenden vier:

$$
\begin{aligned}
& K K^{\prime}+c^{2}+C^{\prime} K+a^{2}=0, \\
& K K^{\prime}+c^{2}-C^{\prime} K^{\prime}+b^{2}=0, \\
& \left(K K^{\prime}+c^{2}\right) \alpha^{\prime}+C K \beta=0, \\
& \left(K K^{\prime}+c^{2}\right) \beta^{\prime}+C K^{\prime} \alpha=0,
\end{aligned}
$$

wenn wir eine fünfte Gleichung beiseite lassen, weil sie sich in die Form $K^{\prime} \alpha^{2}-K \beta^{2}=C^{\prime}$ bringen lässt und folglich in den Gleichungen [1] - [3] entJalten ist.

Durch die sieben Gleichungen $[1]-[3],\left[4^{\prime}\right]-\left[7^{\prime}\right]$ wird unsere Frage nach 
der Möglichkeit einer Geradenschar (41) als $S^{\prime}$ - Schar des Hyperboloides (18) leicht erledigt.

Die Gleichungen [ $\left.b^{\prime}\right],\left[7^{\prime}\right]$ liefern uns $\alpha, \beta$ als Integrale der Gleichung

$$
\left(K K^{\prime}+c^{2}\right)^{2} \alpha^{\prime \prime}-C^{2} K K^{\prime} \alpha=0 \text {, }
$$

und weil für $v$ keine besondere Voraussetzung gegeben ist, können wir dann annehmen

aber dann auch

$$
\alpha=A \sin u, \quad A \text { konst., }
$$

$$
C^{2} K K^{\prime \prime}+\left(K K^{\prime \prime}+c^{2}\right)^{2}=0
$$

Aus $\left[6^{\prime}\right]$ kommt nun

$$
\beta=-A \sqrt{\frac{K^{\prime}}{K}} \cos \mu
$$

und wenn wir hernach $C, K, K^{\prime}$ durch drei konstante Grössen $\alpha^{\prime}, b^{\prime}, c^{\prime}$ ersetzen vermittelst der Formeln

$$
K=-\frac{a^{\prime} b^{\prime} c^{\prime}}{b^{\prime 2}}, \quad K^{\prime}=\frac{\alpha^{\prime} b^{\prime} c^{\prime}}{a^{\prime 2}}, \quad C=\frac{c^{2}-c^{\prime 2}}{c^{\prime}},
$$

die letztere eine Folge der zwei rorangehenden und der Formel $\left(a^{\prime}\right)$, so können wir aus [1] und [3] schliessen:

$$
\gamma=A c^{\prime} \cos u, \quad \delta=A \frac{b^{\prime} c^{\prime}}{a^{\prime}} \sin \mu,
$$

und aus [2]:

$$
C^{\prime}=A^{2} \frac{b^{\prime} c^{\prime}}{a^{\prime}}
$$

Nun bleibt nur noch übrig, auch die Gleichungen [4'], [5'] zu befriedigen. Sie ergeben

$$
c^{2}-c^{\prime 2}+a^{2}-A^{2} c^{\prime 2}=0, \quad c^{2}-c^{\prime 2}+b^{2}-A^{2} c^{\prime 2} \frac{b^{\prime 2}}{a^{\prime 2}}=0
$$

weshalb:

$$
A c^{\prime}=\sqrt{a^{2}+c^{2}-c^{\prime 2}}, \quad A \frac{b^{\prime}}{a^{\prime}} c^{\prime}=\sqrt{b^{2}+c^{2}-c^{\prime 2}} .
$$

Unsere Untersuchung ist hiermit zu Ende gefuhrt. Mit den Ausdrücken $(a),(b)$, (c) für $\alpha, \beta, \gamma, \delta$, dabei $A, a^{\prime}, b^{\prime}, c^{\prime}$ Konstante bezeichnend, die nur den Bedingungen $\left(c^{\prime \prime}\right)$ unterliegen, und mit der in $(b)$ steckenden Quadrat- 


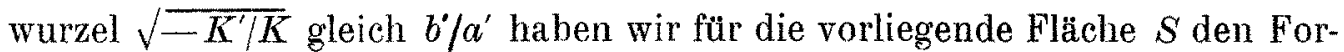
derungen (39) und (40) genügt. Wir sind nun zu folgender Geradenschar gelangt:

$$
\left.\begin{array}{l}
x^{\prime}=z^{\prime} \sin \mu \cdot \frac{\sqrt{a^{2}+c^{2}-c^{\prime 2}}}{c^{\prime}}+\sqrt{a^{2}+c^{2}-c^{\prime 2}} \cos \mu, \\
y^{\prime}=-z^{\prime} \cos \mu . \frac{\sqrt{b^{2}+c^{2}-c^{\prime 2}}}{c^{\prime}}+\sqrt{b^{2}+c^{2}-c^{\prime 2}} \sin u,
\end{array}\right\}
$$

als zu der einzigen möglicluen $S^{\prime}$-Schar von der Form (26). Als Fläche $S$ hatten wir das Hyperboloid

$$
\frac{x^{2}}{a^{2}}+\frac{y^{2}}{b^{2}}-\frac{z^{2}}{c^{2}}=1
$$

gewählt. Die Geradenschar (47) stellt eine Schar von Erzeugenden des konfokalen Hyperboloides

$$
\frac{x^{2}}{a^{2}+\left(c^{2}-c^{\prime 2}\right)}+\frac{y^{2}}{b^{2}+\left(c^{2}-c^{22}\right)}-\frac{z^{2}}{c^{2}-\left(c^{2}-c^{\prime 2}\right)}=1
$$

dar; wir sind deshalb durch das Vorangehende zu keiner anderen Transformation der auf das Hyperboloid abwickelbaren Flächen gekommen als der von Bianchi, die von irgend einer auf eine Fläche zweiter Ordnung abwickelbaren Fläche zu unendlich vielen neuen ebenfalls darauf abwickelbaren Flächen führt. 